\title{
Discordance of community and hospital ultrasound reports for urological abnormalities
}

\author{
Doreen E. Chung, MD; ${ }^{*}$ Tal Platzker; ${ }^{\dagger}$ Justin Chung; ${ }^{\dagger}$ Sidney B. Radomski, $M D^{\dagger}$
}

See related article on page 255

\begin{abstract}
Introduction: In Ontario, community ultrasound clinics do not require the onsite presence of a radiologist at the time of imaging. In hospitals, a radiologist is always present for this step. We compared the discrepancy rate of community and hospital ultrasound reports in a urology practice.
\end{abstract}

Materials and Methods: We retrospectively reviewed the charts of patients who had abdominal ultrasound performed in the community and in the hospital between April 1, 2001 and June 30, 2005. Reports were examined for indication, findings and whether there was a discrepancy. A medical student, a resident, and a staff physician each independently reviewed the findings.

Results: One hundred and twenty-two patients had abdominal ultrasound performed in the community and were followed up with hospital imaging. Sixtynine patients had a community ultrasound followed by hospital ultrasound, with a discordance rate of $52.2 \%$. Fifty-three patients had a community ultrasound and then a hospital CT scan, with a discordance rate of $43.3 \%$. Of patients with discordant ultrasound reports, 23 had an additional CT scan in which all findings were consistent with the hospital ultrasound findings.

Discussion: We found a very high discordance rate between community and hospital ultrasound reports. This is consistent with a study showing that the active role of the radiologist in ultrasound imaging is very important for accurate reporting.

Conclusion: Ultrasound reports form the community in patients referred to our urology practice have a high discrepancy rate when compared with ultrasound or CT scan reports from the hospital setting. We found no discordance between ultrasound and CT scan findings from imaging performed in hospital. We recommend standardization of abdominal ultrasound imaging protocols across Canada to include the presence of a radiologist at all times.

CUAJ 2007;1(3):251-4

\section{Introduction}

Many patients are referred to a urologist after a family physician detects abnormal findings on an abdominal ultrasound report. In Ontario, many ultrasound studies are performed in diagnostic imaging clinics outside of hospitals, sometimes without the physical presence of a radiologist at the time of imaging. In some provinces, such as Alberta and Quebec, it is mandated that a radiologist be on site at all times during the provision of ultrasound. ${ }^{1}$ Conversely, in Ontario, according to the 2006 Clinical Practice Parameters and Facility Standards for Diagnostic Radiology from the College of Physicians and Surgeons of Ontario (CPSO), a radiologist must be available but not on site at the time of the ultrasound examination. The document reads, "It is recognized however that the geographic realities in Canada do not permit the presence of an on-site sonologist in all locations. Adequate documentation of each examination is critical. The imaging physician must be available by telephone to consult with the technologist/sonographer and the referring physician."2

A prospective study of 1510 consecutive patients who underwent ultrasound at a hospital demonstrated that constant physician presence is essential to optimize the provision of a complete and accurate report, especially in the setting of a new diagnosis. ${ }^{3}$ Accurate ultrasound reporting is integral to high-quality patient care because spurious findings of pathology on ultrasound have great psychological impact on patients and lead to unnecessary investigations. Similarly, missed pathology significantly alters the quality of patient outcomes.

In our hospital, a university teaching centre, the radiologist is always present during ultrasound imaging. If the technologist observes any images of uncertain importance, the radiologist can immediately rescan the specific area as well as speak to the patient to obtain further clinical history.

We hypothesized that ultrasound reports from the community have a higher rate of reporting error. We wanted to compare the inconsistencies in findings of community and hospital ultrasound reports in a typical urology referral practice. Our secondary objective was to compare the discrepancy rate between community ultrasound and hospital 
CT scan reports to further evaluate differences in reporting error.

\section{Materials and Methods}

We retrospectively reviewed the charts of all patients who had an ultrasound of the abdomen and pelvis performed in the community between April 1, 2001 and June 30, 2005 and who were referred to our clinic at the Toronto Western Hospital. Patients who did not have subsequent hospital imaging were excluded. We examined radiology reports for indication, findings (i.e., stones, hydronephrosis, cysts, etc.) and whether there was a discrepancy between the community and the hospital report. A medical student, a resident, and a urology staff physician independently reviewed the qualitative findings to determine if there was a discrepancy between the community ultrasound and the hospital ultrasound or CT scan reports. Discrepancies were tabulated and proportions calculated. They were classified as false positive or false negatives in relation to the hospital radiology report. The mean patient age was 55.7 years (range 20-85). Of study subjects, 55 were men and 67 were women. We defined significant findings as those that could cause or progress to clinical symptoms such as stones, tumours and hydronephrosis.

\section{Results}

In total, 122 patients had abdominal ultrasound performed in the community as well as followup imaging performed in the hospital. We were able to compare a community ultrasound report with a follow-up hospital ultrasound report from our institution in 69 patients. We compared community ultrasound reports with follow-up hospital CT scan reports from our institution for 53 patients. Of the 69 patients who had both a community and a hospital ultrasound, 23 also had a hospital CT scan. The CT scan findings were typically used as a "tie-breaker" (in other words, to decide which report findings were accurate when a discrepancy existed between the ultrasound reports). Initial indications to order an ultrasound study included flank pain $(45.1 \%)$, gross or microscopic hematuria $(23 \%)$, lower urinary tract symptoms such as frequency and urgency $(9.8 \%)$, follow-up of renal mass or complex cyst $(7.4 \%)$, recurrent urinary tract infections $(4.1 \%)$ and other (e.g., follow-up of renal calculi and elevated creatinine) (10.7\%).

Of the 69 community ultrasound reports we compared with hospital ultrasound reports, only 33 were found to have identical findings (Table 1); hence, there was a discordance rate of $52.2 \%$. Of the 53 community ultrasound reports we compared with the hospital CT scan reports, only 30 were found to have identical findings, and the discordance rate was $43.3 \%$ (Table 1 ). Of the 36 patients whose hospital and community ultrasound reports contained discrepancies, 23 patients also had CT scans. CT scan findings for all of these patients corresponded with the hospital ultrasound report. The discordance rate between hospital ultrasound and hospital CT scan reports was $0 \%$. In total, the discrepancy rate between hospital and community studies was $48.4 \%$.

Table 2 outlines discordant findings between community and hospital ultrasound reports. The most common false positive findings were hydronephrosis, renal mass, stone and complex cyst. Parapelvic cysts were often mistaken for

\begin{tabular}{lccc}
\hline Table 1: Discordance rates & & \\
\hline $\begin{array}{l}\text { Concordant } \\
\text { Comparison }\end{array}$ & $\begin{array}{c}\text { Discordant } \\
\text { reports, } \\
\text { no. }\end{array}$ & $\begin{array}{c}\text { Discordance } \\
\text { rate, } \%\end{array}$ \\
\hline $\begin{array}{l}\text { Community ultra- } \\
\text { sound v. hospital } \\
\text { ultrasound ( } n=69)\end{array}$ & 33 & 36 & 52.2 \\
$\begin{array}{l}\text { Hospital ultrasound } \\
\text { v. community }\end{array}$ & 23 & 0 & 0 \\
$\begin{array}{l}\text { CT scan ( } n=23 / 69) \\
\text { Community ultra- } \\
\text { sound v. hospital CT } \\
\text { scan }(n=53)\end{array}$ & 30 & 23 & \\
\hline
\end{tabular}


hydronephrosis. Complex cysts were sometimes found to actually be simple cysts. Stones that were found on community ultrasound were not seen on hospital ultrasound. In some patients more than one discrepancy of findings was found. The false negative findings that we encountered most often between ultrasound reports were complex cyst, cortical thinning and renal mass. One patient who was reported to have bilateral renal calculi on a community ultrasound report had bilateral renal masses on hospital ultrasound and CT scan. Some patients who were previously thought to have simple renal cysts had complex cysts on hospital imaging. The proportion of discordant findings that were false positives was $69.8 \%$. The proportion of discrepancies that were false negatives was $30.2 \%$.

Of the discrepancies found between community ultrasound and hospital CT scan reports, 73\% were classified as false positives and $26.9 \%$ were considered false negatives. The most common false positive finding was stone, followed by complex cyst and renal mass. Stones were also the most frequently encountered false negative finding, followed by renal mass. Table 3 describes discordant

\begin{tabular}{|c|c|c|}
\hline Finding & $\begin{array}{c}\text { False } \\
\text { positives, } \\
\text { no. (and \%) }\end{array}$ & $\begin{array}{c}\text { False } \\
\text { negatives, } \\
\text { no. (and \%) }\end{array}$ \\
\hline Hydronephrosis & 17 (39.5) & $2(4.7)$ \\
\hline Stone & $3(7.0)$ & $2(4.7)$ \\
\hline Renal mass & 5 (11.6) & $3(7.0)$ \\
\hline Complex cyst & $3(7.0)$ & $3(7.0)$ \\
\hline Bladder mass & $2(4.7)$ & $0(0.0)$ \\
\hline Cortical thinning & $0(0.0)$ & $3(7.0)$ \\
\hline Total errors & $30(69.8)$ & $13(30.2)$ \\
\hline
\end{tabular}

\begin{tabular}{|c|c|c|}
\hline Finding & $\begin{array}{c}\text { False } \\
\text { positives, } \\
\text { no. (and \%) }\end{array}$ & $\begin{array}{c}\text { False } \\
\text { negatives, } \\
\text { no. (and \%) }\end{array}$ \\
\hline Complex cyst & $6(23.1)$ & $0(0.0)$ \\
\hline Stone & 5 (19.2) & 5 (19.2) \\
\hline Renal mass & $4(15.4)$ & $2(7.7)$ \\
\hline Hydronephrosis & $3(11.5)$ & $0(0.0)$ \\
\hline Other & 1 (3.8) & $0(0.0)$ \\
\hline Total errors & $19(73.0)$ & 7 (26.9) \\
\hline
\end{tabular}

findings between community ultrasound and hospital CT scan reports.

\section{Discussion}

We found a very high discordance rate of $52.2 \%$ between community and hospital ultrasound reports. The discordance rate between community ultrasound and hospital CT scan reports was $43.3 \%$. When reviewing all imaging reports we looked only at significant genitourinary findings. For example, a 1-mm stone seen on a CT scan and missed on an ultrasound was not considered significant; however, a stone $8 \mathrm{~mm}$ in size seen on a CT scan and missed on an ultrasound was considered significant. For some urological conditions, such as renal calculi, CT scan is a more accurate diagnostic modality, compared with ultrasound. This was taken into account when each reviewer independently reviewed reports for significant findings. At the start of our review we assumed that an ultrasound performed in our hospital was the gold standard with which to compare community ultrasound. This was supported by our observation that in patients with discordant urological findings on community and hospital ultrasound reports, CT scan findings were concordant with hospital ultrasound findings in all cases.

There are many reasons for discrepancies to exist between hospital and community ultrasound reporting. In the community, a radiologist is not always physically present to either perform or immediately review the ultrasound. Other possible reasons for a high discrepancy rate may be difference in expertise between community and hospital ultrasound technicians. It is also possible that community ultrasound equipment may not be of equal quality, compared with that of a hospital setting. Consistent with the findings of our study, a study by Chan and colleagues demonstrated that when a radiologist was actively involved throughout the entire ultrasound examination, the interpretation was more accurate. ${ }^{3}$ Concordance of ultrasound findings by technologists and physicians was assessed prospectively for examinations of 1510 consecutive patients who underwent ultrasound during regular working hours. In cases in which a major or minor new diagnosis was made from the ultrasound report, concordance rates were only $36 \%$ and $32 \%$, respectively. 
The potential impact of inaccurate ultrasound reports on individual patient care as well as on the health care system as a whole is very profound. Patients may suffer unnecessary anxiety due to incorrect diagnoses or they may receive sub-optimal care secondary to missed or incorrect pathology. There is also an economic cost to society for lost workdays. The health care system is affected by the cost of unnecessary further investigations, such as repeated ultrasound and secondary CT scans as well as those of treating conditions previously overlooked and treated at later stages.

A potential source of error in our study is temporal bias. The hospital radiological studies were obtained after the community ultrasound studies. Hence, some findings may have actually been present on community ultrasound but not on follow-up imaging (i.e., a stone passing). We did not stratify according to the site of community ultrasound studies. There may have been a wide variation in discrepancy rates depending on the community radiology clinic, but this would have been hard to control and evaluate. Finally, our sample size could have been larger for the study to have more power. A prospective study in which each patient had both a hospital ultrasound and CT scan would have been more accurate. Nevertheless, our discrepancy rates of $52.2 \%$ and $43.3 \%$ are astonishingly high.

\section{Conclusions}

Ultrasound reports from the community, in patients referred to our urology clinic, have a high discrep- ancy rate when compared with ultrasound and CT scan reports from the hospital setting. In contrast, we found no discordance between ultrasound and CT scan findings from imaging performed in the hospital setting. This discordance between community and hospital ultrasound imaging studies may be owing to the radiologist's more active role in the hospital setting. Further randomized controlled studies are needed to evaluate this issue. We recommend standardization of abdominal ultrasound imaging protocols across Canada to include the presence of a radiologist at the time of imaging.

From the *Division of Urology, University of Toronto, Sunnybrook Health Sciences Centre, Toronto, Ont., and †Toronto Western Hospital, University Health Network University of Toronto, Toronto, Ont.

This article has been peer reviewed.

Competing interests: None declared.

\section{References}

1. The Advisory Committee on Radiology and Diagnostic Imaging. College of Physicians and Surgeons of Alberta. Diagnostic imaging standards and guidelines. Edmonton: The College; 2005.

2. Bechai N, Garces P, Hartman A, et al. Independent health facilities clinical practice parameters and facility standards: diagnostic imaging. 3rd ed. Toronto: College of Physicians and Surgeons of Ontario; 2006.

3. Chan V, Hanbridge A, Wilson $S$, et al. Case for active physician involvement in ultrasound practice. Radiology 1996;199:555-60.

Correspondence: Dr. Doreen E. Chung, Division of Urology, University of Toronto Sunnybrook Health Sciences Centre, 2075 Bayview Ave., Suite A309, Toronto ON M4N 3M5; fax 416 480-5185; doreen.chung@utoronto.ca 